\title{
Versatility of Platelet Rich Fibrin (PRF) as a Healing Aid in Routine Oral Surgical Procedures
}

\author{
Vatsal Ramwala \\ Career Postgraduate, Department of Oraland Maxillofacial Surgery, Karnavati School of Dentistry, \\ 907/A,Uvarsad, Gandhinagar, Gujarat, India.(382422)
}

\begin{abstract}
:
Objective: To study the use of Platelet Rich Fibrin (PRF) as a healing aid in different routine oral surgical procedures.

Methods: 5 cases of each type of routine oral surgical procedures like intra alveolar molar extraction, trans alveolar extraction of root stump, surgical extraction of $3^{\text {rd }}$ molar, small cyst enucleation and preprosthetic alveoplasty were selected. PRF was put in all the cases and healing and pain were observed. We mainly focused on soft tissue healing as it indirectly creates good environment for hard tissue formation.

Results: Very good healing progress from $1^{\text {st }}$ to $3^{\text {rd }}$ to $7^{\text {th }}$ postop day was observed in almost all types of cases. Pain also gradually reduced or disappeared on $7^{\text {th }}$ postop day.

Conclusion: PRF is autologous material with very good biological healing properties for routine oral surgical procedures.

Practice Implications: PRF is derived from patient's own blood with techno friendly method of centrifugation of blood. It is a cost effective procedure with one time investment of centrifuge machine only which is not so costly too. Being an autologous material there are no chances of immune rejection and it resorbs nicely and at good rate accelerating the healing process. Its use should be promoted rather than using costly synthetic substances.
\end{abstract}

Keywords: Healing, Pain, PRF

\section{Introduction}

Platelet Rich Fibrin (PRF) was first described by Joseph Choukroun in $2001^{1}$. It is considered as a second generation platelet concentrate because it is free of anticoagulants and biochemical modifires ${ }^{2}$. PRF favours the development of microvascularization, guides the epithelial cell migration, carries the cells involved in tissue regeneration and promotes sustained release of growth factors from 1 to 4 weeks and stimulates the environment for wound healing ${ }^{3}$. We studied the use of PRF as a healing aid in routine oral surgical procedures.

\section{Materials and Methods}

Different types of cases of routine oral surgical procedures were selected like 5 cases of molar intra alveolar extraction, 5 cases of transalveolar extraction of root stump, 5 cases of surgical extraction of third molar, 5 cases of small cyst enucleation and 5 cases of preprosthetic alveoplasty. All the cases were systemically healthy patients. Informed consent was obtained from each patient regarding participation in the study. All the procedures were performed under local anaesthesia by the same surgeon. The study design has been approved by institutional ethical committee.

\section{PRF Preparation}

The informed consent for the procedure of PRF preparation has been obtained from all the cases. 10 $\mathrm{ml}$ venous blood was collected and immediately transferred into the sterile test tube. Then according to Choukroun's criteria centrifugation of blood was done at $3000 \mathrm{rpm}$ for 15 minutes. During centrifugation process, when blood gets in contact with the test tube wall, the platelet gets activated leading to initiation of coagulation cascade. After centrifugation, on the basis of sedimentation principle, the resultant product consists of three fractions. The lower fraction contains the red cell corpuscular base and upper fraction contains straw coloured acellular platelet poor plasma (Fig 1). In between these two in the middle fraction, platelet rich fibrin clot is found. It is derived with tissue holding forcep from test tube and put on sterile gauze wetted with normal saline. It is squeezed between two gauze pieces to remove any red corpuscular base attached with it. Gel like 
PRF clot is thus obtained for use (Fig 2). This PRF was put as a healing aid in all different procedures (Fig 3, 4, $5,6,7)$, which resorbs nicely with surrounding tissue and accelerates the healing.

Postoperative healing was measured after $1^{\mathrm{st}}, 3^{\mathrm{rd}}$ and $7^{\text {th }}$ postop days on the basis of healing index by Landry. ${ }^{13}$ We mainly focused on initial soft tissue healing as it indirectly creates good environment for hard tissue healing. Simultaneously pain was determined by Visual Analogue scale (VAS).

Average score for each type of 5 cases were calculated for $1^{\text {st }}, 3^{\text {rd }}$ and $7^{\text {th }}$ postop days. For example in 5 cases of molar intra alveolar extraction, average scores of healing and pain for $1^{\text {st }}$ postopday, for $3^{\text {rd }}$ postop day and for $7^{\text {th }}$ postop day were calculated.. These average scores were plotted on chart.

\section{Healing Index by Landry 13}

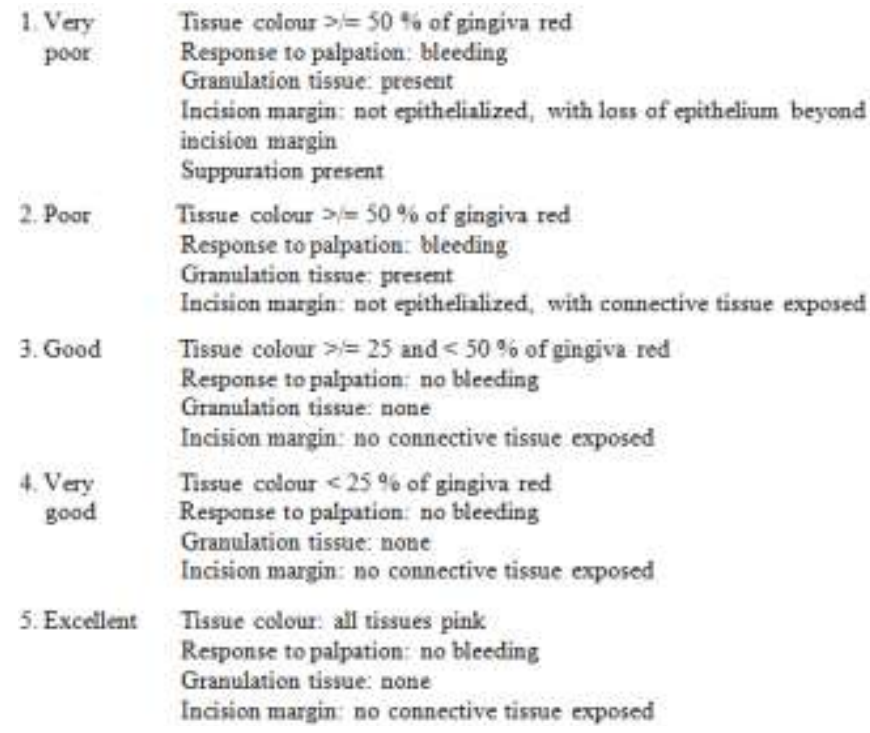

\section{Results}

All the cases were recalled on $1^{\text {st }}, 3^{\text {rd }}$ and $7^{\text {th }}$ postop days. In all the procedures average healing index scores and average VAS numbers were assigned on $1^{\text {st }}, 3^{\text {rd }}$ and $7^{\text {th }}$ postop days and results were depicted on charts (Chart 1 and Chart 2).

\section{Chart 1}

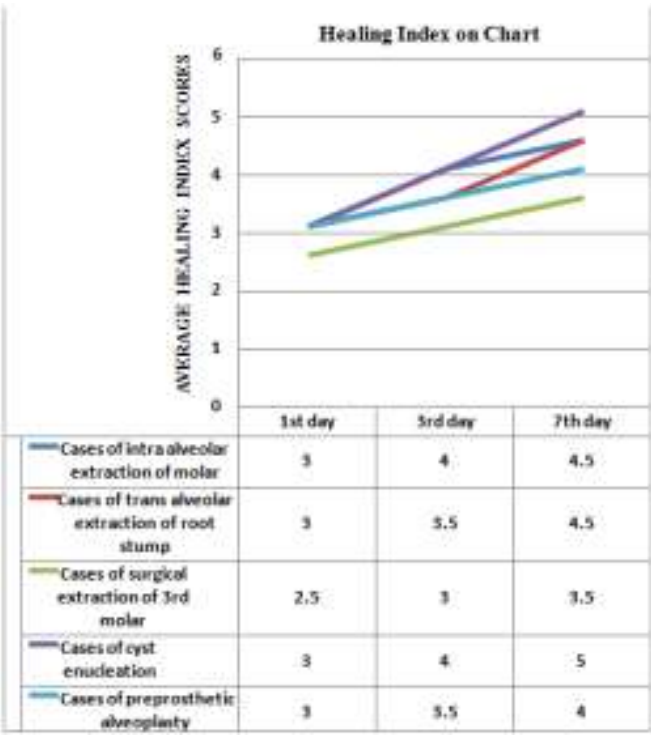


As it shows, on the $7^{\text {th postop }}$ day all the cases progressed to almost very good healing. In the cases of surgical extraction of $3^{\text {rd molar }}$ healing progress on $7^{\text {th }}$ day was little delayed.

Chart 2

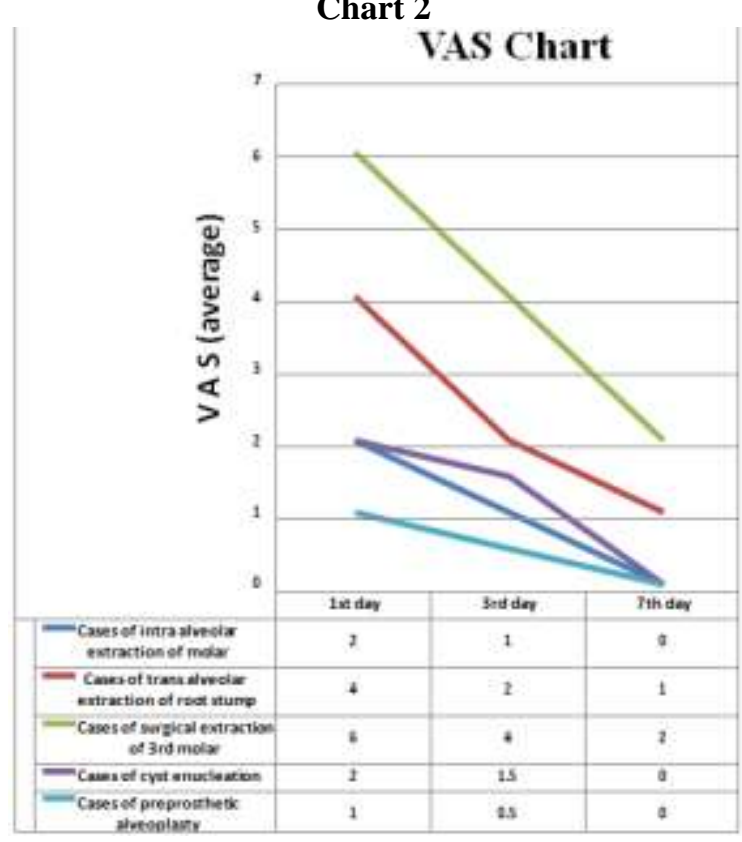

As it shows, there was almost no pain on $7^{\text {th }}$ day in all the types of cases. Mild pain was observed on $7^{\text {th }}$ postop day in the cases of surgical extraction of $3^{\text {rd }}$ molar.

\section{Discussion}

Platelet rich products can be classified as follows according to their leukocyte and fibrin content.

Dohan Ehrenfest classification ${ }^{4}$ :

1. Only platelet rich plasma products

2. Platelet and leukocyte rich plasma products

3. Platelet rich fibrin

4. Platelet and leukocyte rich fibrin

PRF is often called Choukroun's PRF as there are other platelet concentrates with similar names such as Vivostat PRF (considered a pure platelet rich plasma) or Fibrinet (without leukocytes) ${ }^{2}$.

Choukroun's PRF is a leukocyte and platelet rich fibrin biomaterial, which is considered as a second generation platelet concentrate as it is prepared as a natural concentrate without the addition of any anticoagulants. PRF has many advantages over platelet rich plasma (PRP), which is the first generation platelet concentrate. The advantages are ease of preparation, ease of application, minimal expense and lack of biochemical modification (no bovine thrombin or anticoagulant required). This considerably reduces the biochemical handling of blood as well as risks associated with bovine thrombin like antigen-antibody reaction $^{2,5}$. PRF is a strict autologous preparation, without chances of immune rejection.

PRF has been shown to enhance soft tissue healing which indirectly creates a better environment for bone growth ${ }^{10}$. In our five different cases we checked clinically the soft tissue healing effects of PRF. As discussed above, PRF has great healing influence and immune properties; we found in all different five cases that healing was very good on $7^{\text {th }}$ postop day (Chart 1) without any signs of infections. We also measured the pain. In all the cases there was a gradual decrease in pain from $1^{\text {st }}$ to $7^{\text {th }}$ postop day. Only in case of surgical extraction of $3^{\text {rd }}$ molar, pain scale was little high from $1^{\text {st }}$ to $7^{\text {th }}$ day (Chart 2). Singh et al in their study found that there was no statistically significant effect of PRF in reduction of pain after $3^{\text {rd }}$ molar removal $^{9}$. 
Uyanlk et al found in their study that PRF significantly reduced pain after $3^{\text {rd }}$ molar removal ${ }^{6}$. Gaurav Vidhale et al concluded the good healing features in the management of radicular cyst using PRF and bone graft ${ }^{11}$. In our case of radicular cyst, after enucleation, we put PRF in cystic cavity and found very good healing progress from $1^{\text {st }}$ to $7^{\text {th }}$ day. Saluja et al mentioned that cavities filled with PRF showed complete healing in half the time as compared to time required for physiologic healing 5 .

PRF has a dense fibrin network with leukocytes. Leukocytes play an important role in growth factor release, immune regulation, anti infectious activities and matrix remodelling during healing 2 . Leukocytes enmeshed within the PRF releases cytokines like interlukin $1 \beta$ (IL-1 $\beta$ ), IL-4, IL-6 and tumor necrosis factor $\alpha^{6}$. Growth factors contained within the $\alpha$ granules of platelets mainly are Platelet derived growth factors (PDGF), Transforming growth factors- $\beta$ (TGF- $\beta$ ) and Vascular Endothelial Growth Factors (VEGF) along with molecules like fibrinogen, fibronectin and vitronectin ${ }^{7}$.

The natural slow polymerization on contact with taste tube walls results in physiologic thrombin concentration in $\mathrm{PRF}^{8}$. Because of the small blood sample, the quantity of physiologic thrombin is also going to be small in the particular blood sample. Thus conversion of fibrinogen to fibrin takes place slowly ${ }^{5}$. Such slow polymerization results in increased incorporation of the circulating cytokines in the fibrin meshes (intrinsic cytokines). These intrinsic cytokines will be having an increased lifespan and they will released and used only at the time of initial cicatricial matrix remodelling which creates a long term effect ${ }^{2}$. This imparts the significant retro control effects from cytokines on inflammatory regulation ${ }^{8}$.

During gelling of the fibrin structure, the fibrin fibrillae can be assembled in 2 ways, either bilateral junctions or equilateral junctions ${ }^{2}$. PRF has equilateral junctions or 3-D trimolecular network that allows the establishment of a fine and flexible fibrin network able to support cytokines enmeshment and cellular migration. 3-D structure also gives elasticity and flexibility to the PRF membrane ${ }^{8}$.

The natural fibrin network in PRF protects the growth factors from proteolysis. This fibrin structure also entraps the circulating stem cells. PRF also helps in facilitating adhesions and spreading of cells, regulates gene expression of growth factors, growth factor receptors, proteins and determines the outcome of a cell's response to growth factors due to the presence of collagen, fibronectin, elastin, other non collagenous proteins and proteoglycan in the extracellular matrix of $\mathrm{PRF}^{2}$.

The slow polymerization process of PRF promotes the gradual release of growth factors which is ideal for neoangiogenesis. Such growth factors are Basic fibroblast growth factors (FGFb), VEGF and PDGF, which are involved in angiogenic process and useful as chemotactic factors, favouring diapedesis of white blood cells. The fibrin induces the expression of $\alpha \mathrm{v}-\beta 3$ integrin by endothelial cells, allowing the links with structural proteins, such as fibronectin and vitronectin, supporting the process of formation of capillaries ${ }^{7}$.

The fibrin and fibrinogen degradation products (FDP) of PRF stimulate the migration of neutrophils to endothelium and increase the membrane's expression of Cluster of Differentiation CD11c/CD18 receptor. This receptor permits adhesion of the neutrophils to endothelium and fibrinogen as well as the transmigration of neutrophils. Moreover, phagocytosis and enzymatic degradation process are modulated by FDP and hence fibrin also plays an important role in immunity control ${ }^{9}$.

In surgical procedures PRF could serve as a resorbable membrane for guided bone regeneration (GBR), preventing the migration of non desirable cells into bone defect and providing the space that allows the immigration of osteogenic and angiogenic cells and permits the underlying blood clot to mineralize. When mixed with bone graft it may act as a "biological connector", which attracts stem cell, favours the migration of osteoprogenitor cells to the centre of the graft ${ }^{3}$.

Mustafa Tunha et al mentioned that the glass evacuated blood collection tubes used for PRF preparation promote unavoidable silica contact. The silica particles in the tube, although dens enough to sediment with red blood cells, are small enough for a fraction to remain colloidally suspended in the buffy coat, fibrin and platelet poor plasma layers; so, these particles might reach the patient when the product is used for treatment. So they used a Titanium tube which is well established biocompatible material instead of glass or glass coated plastic tubes. They found that Titanium PRF was tightly woven and thicker than classic PRF. This difference may be due to better hemocompatibility of titanium compared to glass, which could have potentially led to the formation of a more polymerized fibrin ${ }^{4}$. 
Eshghpour M et al discussed the various uses of PRF like in soft tissue repair, for osteointegration of implants in sinus lift procedures, in plastic surgery, in cartilage reconstruction, in cardiothoracic surgery, in vascular surgery and to reduce postoperative hematoma ${ }^{12}$.

The PRF has few drawbacks too along with many advantageous properties. Quick handling is very important, as once the blood sample is collected, the natural coagulation process starts immediately in blood and time wasting can lead to diffuse polymerization with small quantity of $\mathrm{PRF}^{2,4,5,8}$. Once the PRF is made, it should be utilized as soon as possible as delaying leads to dehydration, shrinkage and reduced growth factor concentration $^{2}$. PRF cannot be stored because of chances of bacterial contamination ${ }^{2}$ and quantity is less for big surgical defects.

\section{Conclusion}

PRF is versatile healing aid that can be used in routine oral surgical procedures. It acts on all the aspects of healing like angiogenesis, infection prevention, epithelial cover and stem cell activation. It also mechanically protects the wound site. Recently, injectable and advanced forms of PRF are emerging. Being an autologous and cost effective preparation procedure it is an ideal healing aid for developing countries like India. Its use should be promoted in daily practice rather than using costly synthetic substances. It is blood's own Perfection that Raises the Function - P R F!

\section{Conflict of Interest:}

Potential Conflict of Interest Relevant to this Article Does Not Exist.

\section{References}

[1]. Sujata Mohanty, Himani Pathak, Jitender Dabas. Platelet rich fibrin: A new covering material for oral mucosal defects. Journal of oral biology and craniofacial research 2014(4)144-146.

[2]. Preeja C, Arun S. Platelet-rich fibrin: Its role in periodontal regeneration. The Saudi Journal of Dental Research 2014;5:117-122.

[3]. Eduardo Borie, Daniel Garcia, Iara Augusta Orsi et al. Platelet rich fibrin application in dentistry: a literature review. Int J Clin Exp Med 2015;8(5):7922-7929.

[4]. Mustafa Tunha, Hakam Ozdemir, Zafer Kucukodaci et al. A novel platelet concentrate: Titanium prepared platelet rich fibrin. Biomed Research International vol. 2014, Article ID 209548.

[5]. Harish Saluja, Vipin Dehane, Uma Mahindra. Platelet rich fibrin: A second generation platelet concentrate and a new friend of oral and maxillofacial surgeons. Annals of Maxillofacial Surgery January - June 2011; vol 1;issue 1.

[6]. Lokman Onur Uyanik, Kani Bilginaylar, Ilker Etikan. Effects of platelet rich fibrin and piezosurgery on impacted Mandibular third molar surgery outcomes. Head and Face Medicine 2015;11:25.

[7]. Gaetano Marenzi, Francesco Riccitiello, Mariano Tia et al. Influence of leucocyte and platelet rich fibrin (L-PRF) in the healing of simple postextraction sockets: a split mouth study. Biomed Research International Vol. 2015; Article ID 369273.

[8]. Shobha Prakash, Aditi Thakur. Platelet concentrates: Past, present and future. J. Maxillofac. Oral Surg. Jan-Mar 2011:10(1);4549.

[9]. Abhishek Singh, Munish Kohli, Nimish Gupta. Platelet rich fibrin: a novel approach for osseous regeneration. J. Maxillofac. Oral Surg Oct - Dec 2012;11(4):430-434.

[10]. Fernando Suarez-Lopez del Amo, Alberto Monje, Miguel Padial-Molina et al. Biologic agents for periodontal regeneration and implant site development. Biomed Research International Vol. 2015, Article ID 957518.

[11]. Gaurav Vidhale, Deepali Jain, Sourabh Jain et al. Management of Radicular cyst using platelet rich fibrin and iliac bone graft a case report. Journal of clinical and diagnostic research 2015 Jun, Vol-9(6): ZD34-ZD36.

[12]. Majid Eshghpour, Mohamad Reza Majidi, Amir Hossein Nejat. Platelet rich fibrin: An autologous fibrin matrix in surgical procedures: A case report and review literature. Iranian journal of Otorhinolaryngology 2012 vol.24; No.4, Serial No.69.

[13]. Landry RG, Tumbell RS. Effectiveness of benzydamine HCL in treatment of periodontal post-surgical patients. Res Clin Forums 1988;10:105-118.

\section{Figures}

Fig 1 -Threefractionsafter centrifugation ofblood

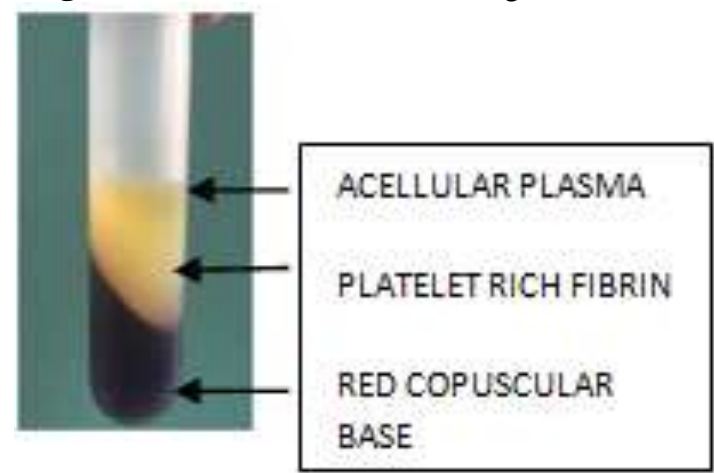

Fig 2 -PRFGel 


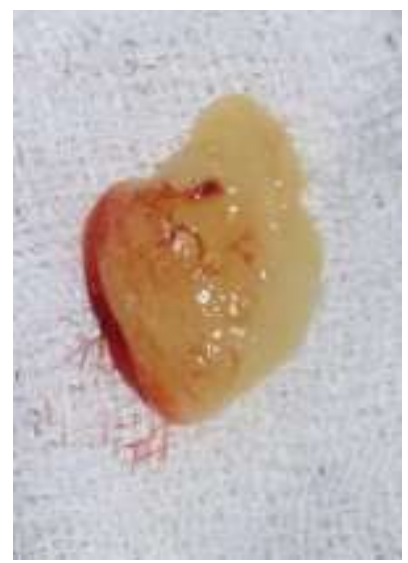

Fig 3 -PRFinmolarextraction socket

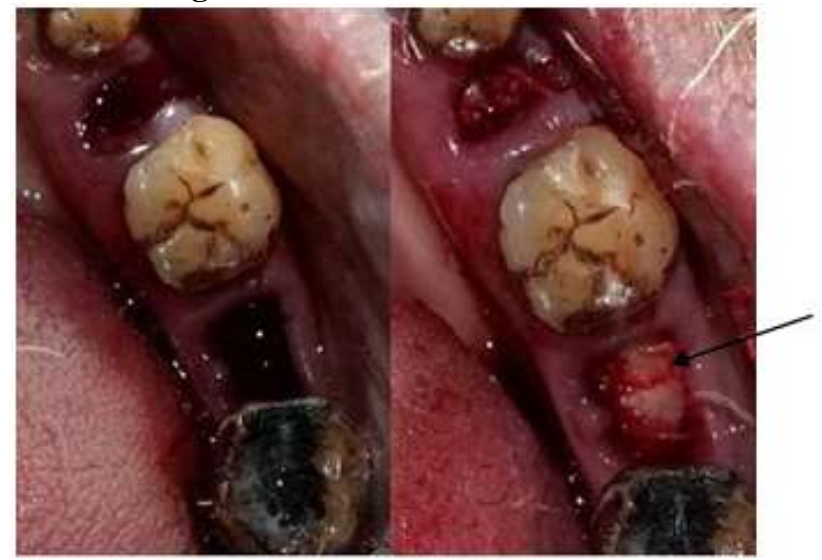

Fig 4 -PRFplacedaftertrans alveolar extractionofroot stump

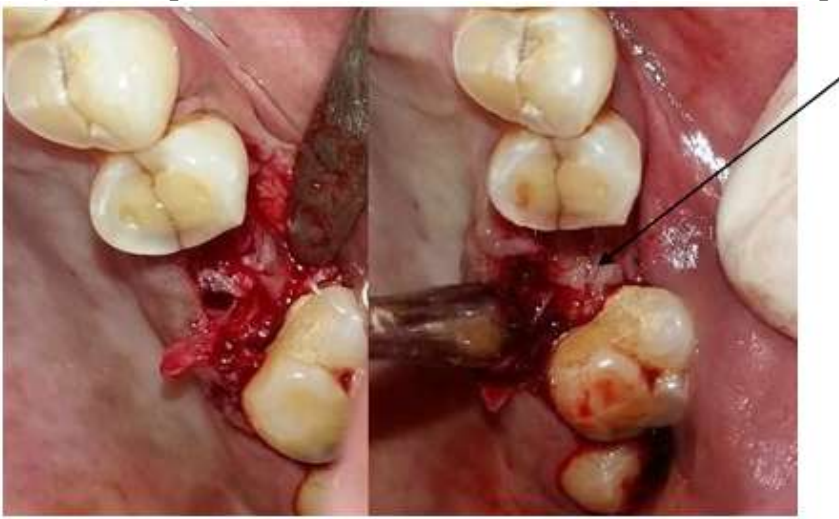

Fig 5 -PRFplacedaftersurgical extraction of $3{ }^{\text {rd }}$ molar

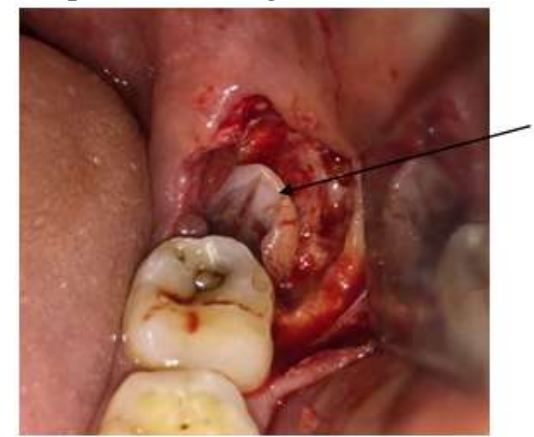


Fig 6 -PRFplacedin cystic cavity

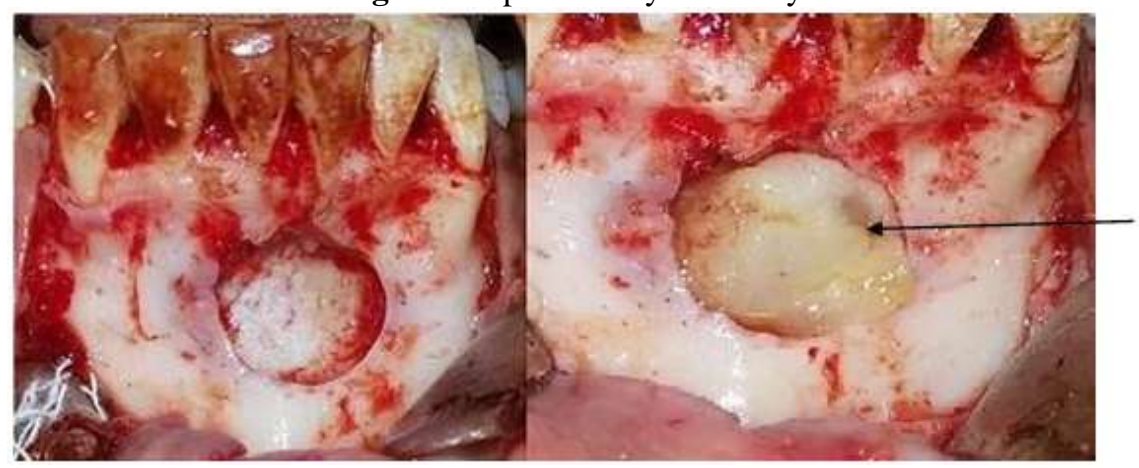

Fig 7 -PRFplacedwithinMucoperiostealflapafterCompletionofAlveoplasty

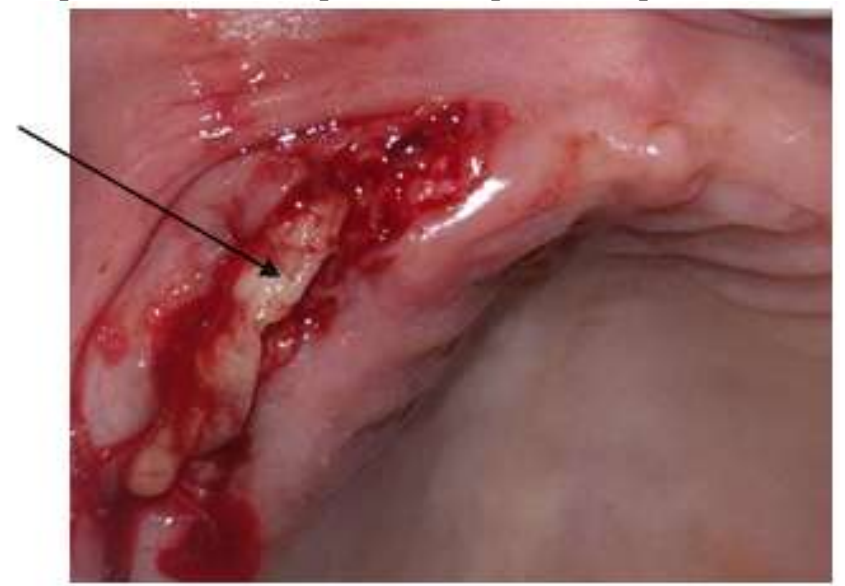

